\title{
Phase I/II study of nivolumab with or without ipilimumab for treatment of recurrent small cell lung cancer (SCLC): CA209-032
}

\author{
Matthew Taylor ${ }^{1 *}$, Scott Antonia ${ }^{2}$, Johanna Bendell ${ }^{3}$, Emiliano Calvo ${ }^{4}$, Dirk Jäger ${ }^{5}$, Filippo de Braud ${ }^{6}$, Patrick A Ott ${ }^{7}$, \\ M Catherine Pietanza ${ }^{8}$, Leora Horn ${ }^{9}$, Dung T Le ${ }^{10}$, Michael A Morse ${ }^{11}$, José A López-Martin ${ }^{12}$, Paolo A Ascierto ${ }^{13}$, \\ Olaf Christensen ${ }^{14}$, Jason S Simon ${ }^{14}$, Chen-Sheng Lin ${ }^{14}$, Joseph Paul Eder ${ }^{15}$
}

From 30th Annual Meeting and Associated Programs of the Society for Immunotherapy of Cancer (SITC 2015) National Harbor, MD, USA. 4-8 November 2015

\section{Background}

Treatment options for SCLC after failing platinum-based (PLT) chemotherapy (CT) are limited. Combined blockade of programmed death-1 (PD-1) and cytotoxic T-lymphocyte antigen-4 (CTLA-4) immune checkpoint pathways has anti-tumor activity with a manageable safety profile. Nivolumab is a fully human IgG4 PD-1 immune checkpoint inhibitor approved for melanoma and squamous NSCLC in the US and for melanoma in the EU and Japan. Interim efficacy and safety of nivolumab +/- ipilimumab, a CTLA-4 checkpoint inhibitor, in pretreated SCLC patients are reported.

\section{Methods}

Patients with progressive disease (PD) after PLT firstline treatment were eligible, regardless of platinum sensitivity, tumor PD-L1 expression, or number of prior CT regimens. Patients were randomized to nivolumab $3 \mathrm{mg} / \mathrm{kg}$ IV Q2W or nivolumab+ipilimumab $(1+1 \mathrm{mg} / \mathrm{kg}$ or $1+3 \mathrm{mg} / \mathrm{kg}$ ) IV Q3W for 4 cycles, followed by nivolumab $3 \mathrm{mg} / \mathrm{kg}$ Q2W. Primary objective was objective response rate (ORR). Additional objectives included safety, progression-free survival (PFS), overall survival (OS), and biomarker analysis.

\section{Results}

Of 90 patients enrolled (nivolumab, $n=40$; nivolumab+ ipilimumab, $\mathrm{n}=50$ [nivolumab $1+$ ipilimumab $1, \mathrm{n}=3$; nivolumab $1+$ ipilimumab $3, \mathrm{n}=47$ ]), $53 \%$ had $\geq 2$ prior regimens. Efficacy results for evaluable patients are

${ }^{1}$ Oregon Health \& Science University, Portland, OR, USA

Full list of author information is available at the end of the article shown (Table 1). $20 \%$ of patients in the nivolumab arm and $42 \%$ in the nivolumab+ipilimumab arms remain on treatment. Discontinuations due to treatment-related adverse events (TRAEs) occurred in $8 \%$ of nivolumab and $11 \%$ of nivolumab+ipilimumab patients. TRAEs (all grades) in $\geq 10 \%$ of patients included fatigue (18\%), diarrhea $(13 \%)$, nausea $(10 \%)$, and decreased appetite $(10 \%)$ with nivolumab; and diarrhea (23\%), fatigue (21\%), rash (21\%), pruritus (19\%), hypothyroidism (15\%), hyperthyroidism (13\%), nausea (13\%), maculopapular rash (13\%), and increased lipase (11\%) with nivolumab 1+ipilimumab 3. Grade 3-4 TRAEs in $\geq 5 \%$ of patients occurred only in the nivolumab $1+$ ipilimumab 3 arm and included diarrhea (9\%) and increased lipase (6\%). Pneumonitis occurred in 2 patients in the nivolumab arm (grade 1-2) and 1 patient in the nivolumab 1+ipilimumab 3 arm (grade 3-4). One patient in the nivolumab $1+$ ipilimumab 3 arm had treatment-related myasthenia gravis with fatal outcome. Updated efficacy, safety, biomarker analysis, and case studies (responses in a patient with PLT-refractory disease and in a patient after crossover to nivolumab/ipilimumab) will be presented.

\section{Conclusions}

In this PD-L1 unselected SCLC population with progression after PLT-CT, nivolumab monotherapy and nivolumab+ipilimumab were generally well tolerated with manageable toxicity. Rare severe toxicities will require close follow-up. Durable responses occurred with nivolumab monotherapy and in combination with ipilimumab. 
Table 1

\begin{tabular}{lcc}
\hline & Nivolumab $(\mathrm{n}-40)$ & ${\text { Nivolumab }+ \text { Ipilimumab }(\mathrm{n}=46)^{\mathrm{a}}}$ \\
\hline ORR, $\%$ & 18 & 17 \\
\hline Complete response, $\%$ & 0 & 2.2 \\
\hline Partial response, $\%$ & 18 & 15 \\
\hline Stable disease, $\%$ & 20 & 37 \\
\hline Disease control rate, $\%$ & 38 & 54 \\
\hline Progressive disease, $\%$ & $53^{\mathrm{c}}$ & 37 \\
\hline Death prior to first response assessment, \% & 10 & $6.5^{\mathrm{d}}$ \\
\hline Not evaluable (no tumor assessment follow-up), \% & 0 & $2.2^{\mathrm{e}}$ \\
\hline Median time to objective response (months) & 1.6 & 2.2 \\
\hline Median DOR, months (95\% Cl) Range & NR 4.1-11+ & \\
\hline DOR & & \\
\hline
\end{tabular}

DOR=duration of response; NR=not reached.

${ }^{\mathrm{a}} 4$ of 50 patients did not reach first tumor assessment at database lock.

${ }^{b}$ Of 17 patients with stage disease in the nivolumab + ipilimumab arm, 7 had confirmed partial response after databse lock, resulting in updated ORR of $32.6 \%$.

${ }^{\mathrm{c}} 1$ patient had progressive disease in the spine, requiring surgery.

${ }^{d} 1$ patient died due to an unrelated adverse event, 1 patient died due to treatment-related myasthenia gravis, 1 patient died due to progressive disease.

${ }^{\mathrm{e}} 1$ patient had an unrelated adverse event leading to permanent discontinuation, and had no post-baseline tumor assessment.

\section{Trial Registry}

Clinical Trial Number: NCT01928394.

\section{Authors' details}

'Oregon Health \& Science University, Portland, OR, USA. ${ }^{2} \mathrm{H}$. Lee Moffitt Cancer Center and Research Institute, Tampa, FL, USA. ${ }^{3}$ Sarah Cannon Research Institute/Tennessee Oncology, PLLC, Nashville, TN, USA. ${ }^{4}$ START Madrid, Centro Integral Oncológico Clara Campa, Madrid, Spain. ${ }^{5}$ Heidelberg University Hospital, Heidelberg, Germany. ${ }^{6}$ Istituto Nazionale dei Tumori, Milan, Italy. ${ }^{7}$ Dana-Farber Cancer Institute, Boston, MA, USA. ${ }^{8}$ Memorial Sloan Kettering Cancer Center, New York, NY, USA. ${ }^{9}$ Vanderbilt-Ingram Cancer Center, Nashville, TN, USA. ${ }^{10}$ The Sidney Kimmel Comprehensive Cancer Center at Johns Hopkins, Baltimore, MD, USA. ${ }^{11}$ Duke University Medical Center, Durham, NC, USA. ${ }^{12}$ Hospital Universitario 12 de Octubre, Madrid, Spain. ${ }^{13}$ Istituto Nazionale Tumori Fondazione G. Pascale, Naples, Italy.

${ }^{14}$ Bristol-Myers Squibb, Princeton, NJ, USA. ${ }^{15}$ Yale Comprehensive Cancer Center, New Haven, CT, USA.

Published: 4 November 2015

doi:10.1186/2051-1426-3-S2-P376

Cite this article as: Taylor et al.: Phase I/II study of nivolumab with or without ipilimumab for treatment of recurrent small cell lung cancer (SCLC): CA209-032. Journal for ImmunoTherapy of Cancer 2015 3(Suppl 2): P376.

\section{Submit your next manuscript to BioMed Central and take full advantage of:}

- Convenient online submission

- Thorough peer review

- No space constraints or color figure charges

- Immediate publication on acceptance

- Inclusion in PubMed, CAS, Scopus and Google Scholar

- Research which is freely available for redistribution

Submit your manuscript at www.biomedcentral.com/submit
Ciomed Central 